\title{
Zero order estimates for functions satisfying generalized functional equations of Mahler type
}

\author{
by \\ Thomas TöPfer (Köln)
}

1. Introduction and results. Zero order estimates for analytic functions are closely related to problems in the theory of transcendental numbers. The basic question, if the value $f(\alpha)$ of a transcendental function $f$ at an algebraic point $\alpha$ is transcendental or - more generally - if the values $f_{1}(\alpha), \ldots, f_{m}(\alpha)$ of several algebraically independent functions $f_{1}, \ldots, f_{m}$ are algebraically independent for algebraic $\alpha$, can be changed into the quantitative problem to give lower bounds for $\left|P\left(f_{1}(\alpha), \ldots, f_{m}(\alpha)\right)\right|$ in terms of the degree and the height of the polynomial $P \in \mathbb{Z}\left[y_{1}, \ldots, y_{m}\right] \backslash\{0\}$, and in general zero order estimates are necessary to solve this problem.

In the case of Mahler functions $f: U_{1}(0) \rightarrow \mathbb{C}$, which satisfy (in the simplest case) a functional equation of the form

$$
f\left(z^{d}\right)=R(z, f(z))
$$

with $d \in \mathbb{N}, d \geq 2$, and a rational function $R(z, y)$, the qualitative and the quantitative question are extensively studied. For a historical survey of the qualitative transcendence results see $[\mathrm{K}],[\mathrm{L}],[\mathrm{LP}]$, and transcendence measures can be found in $[\mathrm{NT}]$ and in the references given there. The first measures for algebraic independence were proved by Becker [B1] and - using a completely different method - by Nesterenko [Ne3]. Both results are effective in the height, but not in the dependence on the degree of the polynomial $P$. This is due to the fact that the construction of the auxiliary function, which is needed in the proof, depends on Siegel's lemma. Since this construction is not explicit, a zero order estimate for the auxiliary function is necessary to derive completely effective measures, and at that time no zero order estimate was available.

Using elementary methods, Wass [W] obtained a zero order estimate and gave an effective version of Nesterenko's result. One year earlier Nish-

1991 Mathematics Subject Classification: Primary 11J91; Secondary 11J81. 
ioka derived the following general zero order estimate, which is much better than Wass' result. The proof was published in [Ni1] and is an extension of Nesterenko's elimination-theoretic method in [Ne1]; more exactly, the method of $[\mathrm{Ne} 2]$ is applied to the polynomial ring $C[z]$ over a field $C$ of characteristic 0 , and applications of this theorem were given by Becker [B2], Nishioka [Ni2], and Töpfer [T1], [T2].

TheOREM (Nishioka [Ni1]). Let $f_{1}, \ldots, f_{m} \in C[[z]]$ be formal power series with coefficients in a field $C$ of characteristic 0 and satisfy

$$
f_{i}\left(z^{d}\right)=\frac{A_{i}\left(z, f_{1}(z), \ldots, f_{m}(z)\right)}{A_{0}\left(z, f_{1}(z), \ldots, f_{m}(z)\right)} \quad(1 \leq i \leq m)
$$

where $d \in \mathbb{N}, d \geq 2$, and $A_{i} \in C\left[z, y_{1}, \ldots, y_{m}\right](0 \leq i \leq m)$ are polynomials with $\operatorname{deg}_{z} A_{i} \leq s$ and $\operatorname{deg}_{y_{1}, \ldots, y_{m}} A_{i} \leq t$. Suppose that $t^{m}<d$ and $Q \in$ $C\left[z, y_{1}, \ldots, y_{m}\right]$ with $\operatorname{deg}_{z} Q \leq M, \operatorname{deg}_{y_{1}, \ldots, y_{m}} Q \leq N$ and $M \geq N \geq 1$. If $Q\left(z, f_{1}(z), \ldots, f_{m}(z)\right) \neq 0$, then

$$
\operatorname{ord}_{0} Q\left(z, f_{1}(z), \ldots, f_{m}(z)\right) \leq c_{0} M N^{m \log d /(\log d-m \log t)},
$$

where $\mu=1+s /(d-t)$ and

$$
\begin{aligned}
& c_{0}=\max \left\{\frac{\operatorname{ord}_{0} A_{0}\left(z, f_{1}(z), \ldots, f_{m}(z)\right)}{d-t},\right. \\
&\left.8 m^{2}(8 d t)^{m} \mu\left(12 m(8 d)^{m-1}\right)^{m \log t /(\log d-m \log t)}\right\} .
\end{aligned}
$$

Recently a more general kind of functional equations was studied by Becker [B3], [B4], [B5]. Suppose that the function $f$ is holomorphic in a neighborhood $U$ of a point $\theta \in \widehat{\mathbb{C}}$, the power series expansion of $f$ at $\theta$ has algebraic coefficients, the transformation $T$ is meromorphic in $U$ and algebraic over the function field $\overline{\mathbb{Q}}(z)$ over the algebraic numbers, and $f$ satisfies a functional equation

$$
A(z, f(z), f(T(z)))=0
$$

for $z \in U$ and a polynomial $A(z, y, w)$ with algebraic coefficients. Under certain assumptions on $f, T, \theta, A$, and $\alpha$ Becker [B4] proved that $f(\alpha)$ is transcendental. Quantitative results for functions which satisfy functional equations of the form (1) with polynomial transformations $T(z) \in \overline{\mathbb{Q}}[z]$ and $A(z, y, w)=w-q(y), q \in \overline{\mathbb{Q}}[z]$ with $\operatorname{deg} q=\operatorname{deg} T$, the so-called Böttcher functions, can be found in [B5].

Qualitative algebraic independence results for certain rational transformations were given by Becker [B3] for functions $f_{1}, \ldots, f_{m}$ satisfying

$$
f_{i}(z)=a_{i}(z) f_{i}(T(z))+b_{i}(z) \quad(1 \leq i \leq m)
$$


with $a_{i}, b_{i} \in \overline{\mathbb{Q}}(z)$ and $T(z)=p\left(z^{-1}\right)^{-1}, p \in \overline{\mathbb{Q}}[z]$ of degree at least 2. In this paper we consider a generalization of (2) and state a zero order estimate which generalizes the above mentioned result of Nishioka. Applications of this result to algebraic independence are given in [T3].

TheOREM 1. Let $f_{1}, \ldots, f_{m} \in C[[z]]$ be formal power series with coefficients in a field $C$ of characteristic 0 and satisfy

$$
f_{i}(T(z))=\frac{A_{i}(z, \underline{f}(z))}{A_{0}(z, \underline{f}(z))} \quad(1 \leq i \leq m),
$$

where $f(z)=\left(f_{1}(z), \ldots, f_{m}(z)\right), T(z)=T_{1}(z) / T_{2}(z)$ is a rational function with $T_{1}, T_{2} \in C[z], d=\max \left\{\operatorname{deg} T_{1}, \operatorname{deg} T_{2}\right\}, \delta=\operatorname{ord}_{0} T(z) \geq 2$, and $A_{i} \in C\left[z, y_{1}, \ldots, y_{m}\right](0 \leq i \leq m)$ are polynomials with $\operatorname{deg}_{z} A_{i} \leq s$ and $\operatorname{deg}_{y_{1}, \ldots, y_{m}} A_{i} \leq t$. Suppose that $t^{m}<\delta$ and $Q \in C\left[z, y_{1}, \ldots, y_{m}\right]$ with $\operatorname{deg}_{z} Q \leq M, \operatorname{deg}_{y_{1}, \ldots, y_{m}} Q \leq N$ and $M \geq N \geq 1$. If $Q(z, \underline{f}(z)) \neq 0$, then

$$
\operatorname{ord}_{0} Q(z, \underline{f}(z)) \leq c_{1} M N^{m \log d /(\log \delta-m \log t)},
$$

where $\mu=1+s /(d-t)$ and

$$
\begin{aligned}
& c_{1}=\max \left\{\frac{\operatorname{ord}_{0} A_{0}(z, \underline{f}(z))}{\delta-t},\right. \\
&\left.\mu d \delta^{-1} m^{2}(8 \delta t)^{m}\left(4 m(8 \delta)^{m-1}\right)^{\log d /(\log \delta-m \log t)-1}\right\} .
\end{aligned}
$$

REMARK. In the special case $T(z)=z^{d}$, we have $\delta=d$, and the assertion of the theorem is just Nishioka's result [Ni1] with a slightly better constant.

Corollary 1. Let $f_{1}, \ldots, f_{m} \in C[[z]]$ be formal power series with coefficients in a field $C$ of characteristic 0 which satisfy

$$
f_{i}(z)=a_{i}(z) f_{i}(T(z))+b_{i}(z) \quad(1 \leq i \leq m),
$$

where $a_{i}, b_{i} \in C(z)$ are rational functions, $T(z)=p\left(z^{-1}\right)^{-1}$ with a polynomial $p \in C[z]$ and $d=\operatorname{deg} p \geq 2$. Suppose that $Q \in C\left[z, y_{1}, \ldots, y_{m}\right]$ with $\operatorname{deg}_{z} Q \leq M, \operatorname{deg}_{y_{1}, \ldots, y_{m}} Q \leq N$ and $M \geq N \geq 1$. If $Q(z, \underline{f}(z)) \neq 0$, then

$$
\operatorname{ord}_{0} Q(z, \underline{f}(z)) \leq c_{1} M N^{m}
$$

with $c_{1}=c_{1}\left(a_{i}, b_{j}, d, m\right) \in \mathbb{R}_{+}$as in Theorem 1 .

Proof. Notice that $d=\operatorname{deg} p=\operatorname{ord}_{0} T=\delta>t=1$.

Corollary 2. Let $f_{1}, \ldots, f_{m} \in C[[z]]$ be formal power series with coefficients in a field $C$ of characteristic 0 which satisfy

$$
f_{i}(z)=a_{i}(z) f_{i}(T(z))+b_{i}(z) \quad(1 \leq i \leq m),
$$

where $a_{i}, b_{i} \in C(z)$ are rational functions and $T \in C[z]$ is a polynomial with $d=\operatorname{deg} T \geq \delta=\operatorname{ord}_{0} T \geq 2$. Suppose that $Q \in C\left[z, y_{1}, \ldots, y_{m}\right]$ with 
$\operatorname{deg}_{z} Q \leq M, \operatorname{deg}_{y_{1}, \ldots, y_{m}} Q \leq N$ and $M \geq N \geq 1$. If $Q(z, \underline{f}(z)) \neq 0$, then

$$
\operatorname{ord}_{0} Q(z, \underline{f}(z)) \leq c_{1} M N^{m \log d / \log \delta}
$$

with $c_{1}=c_{1}\left(a_{i}, b_{j}, d, \delta, m\right) \in \mathbb{R}_{+}$as in Theorem 1 .

The proof of Theorem 1 depends on the following criterion for algebraic independence over fields of Laurent series. This criterion is based on Nishioka's result [Ni1], hence on the elimination-theoretic method of Nesterenko [Ne1], [Ne2] and Philippon [P1], [P2].

For the statement of the criterion we need some notations. Suppose $C$ is a field of characteristic $0, v$ the valuation $\operatorname{ord}_{0}$ of the field $C((z))$ of Laurent series or its unique extension to the algebraic closure $\overline{C((z))}$. For $\underline{\omega} \in \overline{C((z))}^{m}$ put $v(\underline{\omega})=\min _{1 \leq i \leq m}\left\{v\left(\omega_{i}\right)\right\}$, and for polynomials $Q\left(z, y_{0}, y_{1}, \ldots, y_{m}\right) \in$ $C[\underline{y}]$ with

$$
Q(z, \underline{y})=\sum_{\mu_{0}, \ldots, \mu_{m}=0}^{\sigma} q_{\mu_{0}, \ldots, \mu_{m}}(z) y_{0}^{\mu_{0}} \ldots y_{m}^{\mu_{m}}
$$

define

$$
v(Q)=\min _{\mu_{0}, \ldots, \mu_{m}}\left\{v\left(q_{\mu_{0}, \ldots, \mu_{m}}\right)\right\}, \quad N(Q)=\operatorname{deg}_{y_{1}, \ldots, y_{m}} Q, \quad H(Q)=\operatorname{deg}_{z} Q .
$$

Theorem 2. Let $C$ be a field of characteristic 0 and $\underline{\omega} \in \overline{C((z))}^{m}$. Suppose that there exist increasing functions $\Psi_{1}, \Psi_{2}: \mathbb{N} \rightarrow \mathbb{R}_{+}$, positive real numbers $\Phi_{1}, \Phi_{2}, \Lambda$, a nonnegative integer $k_{1}$ and for each $k \in\left\{0, \ldots, k_{1}\right\}$ a set of polynomials $Q_{k}^{(1)}, \ldots, Q_{k}^{\left(n_{k}\right)} \in C\left[z, y_{1}, \ldots, y_{m}\right]$ with the following properties for $k \in\left\{0, \ldots, k_{1}\right\}, i \in\left\{1, \ldots, n_{k}\right\}$ :

(i) $\Phi_{2} \geq \Phi_{1}, \Psi_{2}(k) \geq \max \left\{\Psi_{1}(k),-2 v(\underline{\omega})\right\}, \Lambda \geq \Psi_{2}(k+1) / \Psi_{1}(k)$,

(ii) (a) $N\left(Q_{k}^{(i)}\right) \leq \Phi_{1}$,

(b) $H\left(Q_{k}^{(i)}\right) \leq \Phi_{2}$,

(c) $v\left(Q_{k}^{(i)}(\underline{\omega})\right) \geq \Psi_{1}(k)$,

(d) $v(\underline{\omega}-\underline{\theta}) \leq \Psi_{2}(k)$ for all common zeros $\underline{\theta} \in \overline{C((z))}^{m}$ of $Q_{k}^{(1)}, \ldots$ $\ldots, Q_{k}^{\left(n_{k}\right)}$,

(iii) $\Psi_{1}\left(k_{1}\right)>2 m(4 \Lambda)^{m-1} c_{3} \Phi_{1}^{m-1} \max \left\{\Phi_{1} \Psi_{2}(0), m \Phi_{2}\right\}$, where $c_{3}=1$ for $v(\underline{\omega}) \geq 0$ and $c_{3}=(2 m)^{m}$ for $v(\underline{\omega})<0$.

Then we have with $c_{4}=m$ for $v(\underline{\omega}) \geq 0$ and $c_{4}=2^{m} m^{m+2}$ for $v(\underline{\omega})<0$,

$$
\Psi_{1}\left(k_{1}\right) \leq c_{4}(4 \Lambda)^{m} \Phi_{1}^{m} \Phi_{2} .
$$

2. Notations and lemmas. For polynomials $Q\left(z, y_{0}, y_{1}, \ldots, y_{m}\right) \in R[\underline{y}]$ with $R=C[z]$ let $H(Q), N(Q), v(Q)$ be defined as above. If $I \subset R[y]$ is a homogeneous ideal, then $h(I)$ denotes the height of $I, \operatorname{rad} I$ is the radical of $I$, and $Z(I)$ is the zero set of $I$ in $\overline{C((z))}{ }^{m+1} \backslash\{\underline{0}\}$. For the definition 
of $N(I), H(I)$ (resp. $B(I)$ in $[\mathrm{Ni1}]$ ) and $v(I(\underline{\beta}))$ for $\underline{\beta} \in \overline{C((z))}^{m+1} \backslash\{\underline{0}\}$ the reader is referred to Nishioka's paper [Ni1]. The projective distance of $\beta, \underline{\theta} \in \overline{C((z))}^{m+1} \backslash\{\underline{0}\}$ is defined as

$$
V(\underline{\beta}, \underline{\theta})=-v(\underline{\beta})-v(\underline{\theta})+\min _{0 \leq i, j \leq m}\left\{v\left(\beta_{i} \theta_{j}-\beta_{j} \theta_{i}\right)\right\},
$$

and for homogeneous ideals $I$ put

$$
V(\underline{\beta}, Z(I))=\sup _{\underline{\theta} \in Z(I)}\{V(\underline{\beta}, \underline{\theta})\} .
$$

Lemma 1. Suppose that $P \in R[\underline{y}] \backslash\{0\}$ is a homogeneous polynomial, $I=$ $(P)$ is the principal ideal in $R[\underline{y}]$ generated by $P$, and $\underline{\beta} \in \overline{C((z))}^{m+1} \backslash\{\underline{0}\}$. Then

$$
N(I)=N(P), \quad H(I) \leq H(P), \quad v(I(\underline{\beta})) \geq v(P(\underline{\beta}))-N(P) v(\underline{\beta}) .
$$

Proof. See [Ni1], Proposition 1.

Lemma 2. Suppose that $\underline{\beta} \in \overline{C((z))}^{m+1} \backslash\{\underline{0}\}, I$ is an unmixed homogeneous ideal in $R[y], h(I) \leq m$, and $I=I_{1} \cap \ldots \cap I_{s} \cap I_{s+1} \cap \ldots \cap I_{t}$ is its irreducible primary decomposition with $I_{l} \cap R=(0)$ for $l \leq s$ and $I_{s+1} \cap \ldots \cap I_{t}=(b), b \in R \backslash\{0\}$. For $l \leq s$ let $k_{l}$ be the exponent of the ideal $I_{l}$ and $\mathcal{P}_{l}=\operatorname{rad} I_{l}$. Then

(i) $\sum_{l=1}^{s} k_{l} N\left(\mathcal{P}_{l}\right)=N(I)$,

(ii) $H(b)+\sum_{l=1}^{s} k_{l} H\left(\mathcal{P}_{l}\right)=H(I)$,

(iii) $v(b)+\sum_{l=1}^{s} k_{l} v\left(\mathcal{P}_{l}(\underline{\beta})\right)=v(I(\underline{\beta}))$,

(iv) $0 \leq v(b) \leq H(b) \leq \bar{H}(I)$.

When $s=t$, the terms $H(b)$ and $v(b)$ are missing.

Pr o of. See [Ni1], Proposition 2.

Lemma 3. Suppose that $\beta \in \overline{C((z))}^{m+1} \backslash\{\underline{0}\}, \mathcal{P}$ is a nonzero homogeneous prime ideal of $R[\underline{y}]$ with $\mathcal{P} \cap R=(0)$ and $h(\mathcal{P}) \leq m, Q \in R[\underline{y}]$ is a homogeneous polynomial with $Q \notin \mathcal{P}$ and

$$
\Lambda(v(Q(\underline{\beta}))-v(\underline{\beta}) N(Q)) \geq \min \{X, V(\underline{\beta}, Z(\mathcal{P}))\}>0,
$$

where $v(\mathcal{P}(\beta)) \geq X$ and $\Lambda \geq 1$. If $r=m+1-h(\mathcal{P}) \geq 2$, then there exists an unmixed homogeneous ideal $I \subset R[\underline{y}]$ with $Z(I)=Z(\mathcal{P}, Q), h(I)=m-r+2$, such that

(i) $N(I) \leq N(\mathcal{P}) N(Q)$,

(ii) $H(I) \leq H(\mathcal{P}) N(Q)+N(\mathcal{P}) H(Q)$,

(iii) $v(I(\underline{\omega})) \geq X / \Lambda-H(\mathcal{P}) N(Q)-N(\mathcal{P}) H(Q)$.

If $h(\mathcal{P})=m$, then the right side of inequality (iii) is not positive. 
Proof. If $X \leq V(\underline{\beta}, Z(\mathcal{P}))$, we know

$$
v(Q(\underline{\beta}))-v(\underline{\beta}) N(Q) \geq X / \Lambda,
$$

and Lemma 3 of [Ni1] yields the assertion. If $V(\underline{\beta}, Z(\mathcal{P})) \leq X$, we have

$$
v(Q(\underline{\beta}))-v(\underline{\beta}) N(Q) \geq V(\underline{\beta}, Z(\mathcal{P})) / \Lambda,
$$

and Lemma 4 of [Ni1] implies the assertion.

Lemma 4. Suppose $I \subset R[\underline{y}]$ is a nonzero unmixed homogeneous ideal, $I \cap R=(0)$, and $r=m+1-h(I) \geq 1$. Then for every $\underline{\beta} \in \overline{C((z))}^{m+1} \backslash\{\underline{0}\}$ we have

$$
N(I) V(\underline{\beta}, Z(I)) \geq v(I(\underline{\beta})) / r-2 H(I) .
$$

Proof. See Lemma 6 of [Ni1].

3. Proof of Theorem 2. The proof is analogous to the proof of Theorem 6 in [T1]. As usual in elimination theory, we show by induction that there exist homogeneous prime ideals $\mathcal{P}_{l} \subset R[\underline{y}]$ with $h\left(\mathcal{P}_{l}\right)=l(l=1, \ldots, m)$, which satisfy

$$
\begin{aligned}
N\left(\mathcal{P}_{l}\right) & \leq \Phi_{1}^{l} \\
H\left(\mathcal{P}_{l}\right) & \leq l \Phi_{1}^{l-1} \Phi_{2}, \\
v\left(\mathcal{P}_{l}(\underline{\beta})\right) & \geq \frac{\Psi_{1}\left(k_{1}\right)}{2(4 \Lambda)^{l-1} \Phi_{1}^{l}} N\left(\mathcal{P}_{l}\right)+\frac{\Psi_{1}\left(k_{1}\right)}{2(4 \Lambda)^{l-1} l \Phi_{1}^{l-1} \Phi_{2}} H\left(\mathcal{P}_{l}\right),
\end{aligned}
$$

where $\underline{\beta}=(1, \underline{\omega}) \in \overline{C((z))}^{m+1} \backslash\{\underline{0}\}$ for $\underline{\omega} \in \overline{C((z))}^{m}$ as in Theorem 2 . In the last step for $l=m+1$ Lemma 3 implies the asserted inequality of Theorem 2.

Without loss of generality we may assume that $v(\underline{\omega}) \geq 0$. If $v(\underline{\omega})<0$, we suppose that $v\left(\omega_{1}\right), \ldots, v\left(\omega_{\kappa}\right)<0 \leq v\left(\omega_{\kappa+1}\right), \ldots, v\left(\omega_{m}\right)$ and apply the transformation

$$
\begin{aligned}
& Q\left(y_{1}, \ldots, y_{m}\right) \rightarrow \bar{Q}\left(y_{1}, \ldots, y_{m}\right) \\
& \quad=\left(y_{1} \ldots y_{\kappa}\right)^{\operatorname{deg} Q} Q\left(1 / y_{1}, \ldots, 1 / y_{\kappa}, y_{\kappa+1}, \ldots, y_{m}\right)
\end{aligned}
$$

to all polynomials which occur in the proof. Thus with $\underline{\underline{\omega}}=\left(1 / \omega_{1}, \ldots, 1 / \omega_{\kappa}\right.$, $\left.\omega_{\kappa+1}, \ldots, \omega_{m}\right)$ we have

$$
\begin{gathered}
N(\bar{Q}) \leq m \operatorname{deg} Q \leq m \Phi_{1}=\Phi_{1}^{*}, \quad H(\bar{Q})=H(Q) \leq \Phi_{2} \leq m \Phi_{2}=\Phi_{2}^{*}, \\
v(\bar{Q}(\underline{\bar{\omega}}))=v\left(\left(\omega_{1} \ldots \omega_{\kappa}\right)^{-\operatorname{deg} Q} Q(\underline{\omega})\right) \geq v(Q(\underline{\omega})) \geq \Psi_{1}(k)=\Psi_{1}^{*}(k) .
\end{gathered}
$$

Now we suppose that $\underline{\bar{\theta}}=\left(\bar{\theta}_{1}, \ldots, \bar{\theta}_{m}\right)$ is a common zero of $\bar{Q}_{k}^{(1)}, \ldots, \bar{Q}_{k}^{\left(n_{k}\right)}$. If $\bar{\theta}_{i}=0$ for some $i \in\{1, \ldots, \kappa\}$, then $v(\underline{\underline{\omega}}-\underline{\bar{\theta}}) \leq v\left(\bar{\omega}_{i}\right)=-v\left(\omega_{i}\right) \leq-v(\underline{\omega}) \leq$ 
$\Psi_{2}(k)$; otherwise

$$
\begin{aligned}
v(\underline{\bar{\omega}}-\underline{\bar{\theta}}) & =\min _{\substack{1 \leq i \leq \kappa \\
\kappa+1 \leq j \leq m}}\left\{-v\left(\omega_{i}\right)-v\left(\theta_{i}\right)+v\left(\omega_{i}-\theta_{i}\right), v\left(\omega_{j}-\theta_{j}\right)\right\} \\
& \leq-2 v(\underline{\omega})+v(\underline{\omega}-\underline{\theta}) \leq 2 \Psi_{2}(k)=\Psi_{2}^{*}(k) .
\end{aligned}
$$

Hence (i), (ii) of Theorem 2 are fulfilled with $\Lambda^{*}=2 \Lambda, v(\underline{\underline{\omega}}) \geq 0$, and (iii) follows from

$$
\begin{aligned}
\Psi_{1}^{*}\left(k_{1}\right) & >2 m(4 \Lambda)^{m-1} 2^{m-1}\left(m \Phi_{1}\right)^{m-1} \max \left\{2 m \Phi_{1} \Psi_{2}(0), m \Phi_{2}\right\} \\
& =2 m\left(4 \Lambda^{*}\right)^{m-1} \Phi_{1}^{* m-1} \max \left\{\Phi_{1}^{*} \Psi_{2}^{*}(0), \Phi_{2}^{*}\right\} .
\end{aligned}
$$

Therefore we suppose from now on that all assumptions of Theorem 2 are satisfied with $v(\underline{\omega}) \geq 0$.

Throughout the proof of Theorem 2 let $Q^{*}$ denote the homogenization of the polynomial $Q \in R\left[y_{1}, \ldots, y_{m}\right]$, i.e. $Q^{*} \in R\left[y_{0}, y_{1}, \ldots, y_{m}\right]=$ $R[\underline{y}]$ is homogeneous with $\operatorname{deg}_{\underline{y}} Q^{*}=\operatorname{deg}_{y_{1}, \ldots, y_{m}} Q$ and $Q^{*}\left(1, y_{1}, \ldots, y_{m}\right)=$ $Q\left(y_{1}, \ldots, y_{m}\right)$.

In the first step, $l=1$, we choose one of the polynomials $Q_{k_{1}}^{(1)}, \ldots, Q_{k_{1}}^{\left(n_{k_{1}}\right)}$, say $Q_{k_{1}}^{(1)}$, and define the unmixed homogeneous ideal $I^{(1)}=\left(Q_{k_{1}}^{(1) *}\right) \subset R[\underline{y}]$. Then $h\left(I^{(1)}\right)=1$ and, by Lemma 1 ,

(6) $\quad N\left(I^{(1)}\right) \leq \Phi_{1}, \quad H\left(I^{(1)}\right) \leq \Phi_{2}, \quad v\left(I^{(1)}(\underline{\beta})\right) \geq v\left(Q_{k_{1}}^{(1)}(\underline{\omega})\right) \geq \Psi_{1}\left(k_{1}\right)$.

Now suppose that $\mathcal{P}^{(1)}, \ldots, \mathcal{P}^{(s)} \subset R[\underline{y}]$ are the associated prime ideals of $I^{(1)}$, which are defined in Lemma 2. Then $N\left(\mathcal{P}^{(i)}\right) \leq \Phi_{1}, H\left(\mathcal{P}^{(i)}\right) \leq$ $\Phi_{2}, h\left(\mathcal{P}^{(i)}\right)=1$ for $i=1, \ldots, s$. If none of the prime ideals $\mathcal{P}^{(i)}$ satisfies inequality (5), we have

$$
v\left(\mathcal{P}^{(i)}(\underline{\beta})\right)<\frac{\Psi_{1}\left(k_{1}\right)}{2 \Phi_{1}} N\left(\mathcal{P}^{(i)}\right)+\frac{\Psi_{1}\left(k_{1}\right)}{2 \Phi_{2}} H\left(\mathcal{P}^{(i)}\right)
$$

for $i=1, \ldots, s$, and Lemma 2(iii), (iv) together with Theorem 2(iii) implies $v\left(I^{(1)}(\underline{\beta})\right)<v(b)+\frac{\Psi_{1}\left(k_{1}\right)}{2 \Phi_{1}} \sum_{i=1}^{s} k_{i} N\left(\mathcal{P}^{(i)}\right)+\frac{\Psi_{1}\left(k_{1}\right)}{2 \Phi_{2}} \sum_{i=1}^{s} k_{i} H\left(\mathcal{P}^{(i)}\right) \leq \Psi_{1}\left(k_{1}\right)$,

but this contradicts the rightmost inequality of (6). Thus at least one prime ideal, say $\mathcal{P}^{(1)}$, satisfies (3)-(5), and we define $\mathcal{P}_{1}=\mathcal{P}^{(1)}$.

Now we assume that (3)-(5) are fulfilled for $l-1$ with $l \in\{2, \ldots, m\}$. With

$$
X=\frac{\Psi_{1}\left(k_{1}\right)}{2(4 \Lambda)^{l-2} \Phi_{1}^{l-1}} N\left(\mathcal{P}_{l-1}\right)+\frac{\Psi_{1}\left(k_{1}\right)}{2(4 \Lambda)^{l-2}(l-1) \Phi_{1}^{l-2} \Phi_{2}} H\left(\mathcal{P}_{l-1}\right)
$$

the inequalities $v\left(\mathcal{P}_{l-1}(\underline{\beta})\right) \geq X>\Psi_{2}(0)$ hold, the latter by Theorem 2(iii). 
Furthermore Lemma 4 and Theorem 2(iii) imply

$$
V\left(\underline{\beta}, Z\left(\mathcal{P}_{l-1}\right)\right) \geq \frac{X}{(m+1-(l-1)) N\left(\mathcal{P}_{l-1}\right)}-2 \frac{H\left(\mathcal{P}_{l-1}\right)}{N\left(\mathcal{P}_{l-1}\right)}>\Psi_{2}(0) .
$$

Since

$$
X \leq \Psi_{1}\left(k_{1}\right)\left(\frac{1}{2(4 \Lambda)^{l-2}}+\frac{1}{2(4 \Lambda)^{l-2}(l-1)}\right) \leq \Psi_{1}\left(k_{1}\right) \leq \Psi_{2}\left(k_{1}\right),
$$

there exists a number $k_{l} \in\left\{0, \ldots, k_{1}\right\}$ with

$$
\Psi_{2}\left(k_{l}\right)<\min \left\{X, V\left(\underline{\beta}, Z\left(\mathcal{P}_{l-1}\right)\right)\right\} \leq \Psi_{2}\left(k_{l}+1\right) .
$$

We claim that at least one of the polynomials $Q_{k_{l}}^{(1) *}, \ldots, Q_{k_{l}}^{\left(n_{k_{l}}\right) *}$ does not belong to $\mathcal{P}_{l-1}$. Otherwise $Z\left(\mathcal{P}_{l-1}\right) \subset Z\left(Q_{k_{l}}^{(1) *}, \ldots, Q_{k_{l}}^{\left(n_{k_{l}}\right) *}\right)$, and then Theorem 2(ii)(d) implies after some calculation

$$
\Psi_{2}\left(k_{l}\right)<V\left(\underline{\beta}, Z\left(\mathcal{P}_{l-1}\right)\right) \leq V\left(\underline{\beta}, Z\left(Q_{k_{l}}^{(1) *}, \ldots, Q_{k_{l}}^{\left(n_{k_{l}}\right) *}\right)\right) \leq \Psi_{2}\left(k_{l}\right),
$$

but this is a contradiction. Without loss of generality we may assume that $Q_{k_{l}}^{(1) *} \notin \mathcal{P}_{l-1}$.

Define $\sigma \in \mathbb{R}_{+}$by

$$
\min \left\{X, V\left(\underline{\beta}, Z\left(\mathcal{P}_{l-1}\right)\right)\right\}=\sigma v\left(Q_{k_{l}}^{(1) *}(\underline{\beta})\right)=\sigma v\left(Q_{k_{l}}^{(1)}(\underline{\omega})\right) .
$$

From Theorem 2(i), (ii)(c) and the choice of $k_{l}$ we get

$$
\sigma \Psi_{1}\left(k_{l}\right) \leq \sigma v\left(Q_{k_{l}}^{(1)}(\underline{\omega})\right) \leq \Psi_{2}\left(k_{l}+1\right) \leq \Lambda \Psi_{1}\left(k_{l}\right),
$$

hence $\sigma \leq \Lambda$ and

$$
\Lambda v\left(Q_{k_{l}}^{(1) *}(\underline{\beta})\right) \geq \min \left\{X, V\left(\underline{\beta}, Z\left(\mathcal{P}_{l-1}\right)\right)\right\}
$$

with $\Lambda \geq 1$ (notice that $v(\underline{\beta})=v(1)=0$ ). By Lemma 3 and Theorem 2(ii), (iii) there exists an unmixed homogeneous ideal $I^{(l)} \subset R[\underline{y}]$ with $h\left(I^{(l)}\right)=l$ and

$$
\begin{aligned}
& N\left(I^{(l)}\right) \leq \Phi_{1} N\left(\mathcal{P}_{l-1}\right) \leq \Phi_{1}^{l}, \\
& H\left(I^{(l)}\right) \leq \Phi_{1} H\left(\mathcal{P}_{l-1}\right)+\Phi_{2} N\left(\mathcal{P}_{l-1}\right) \leq l \Phi_{1}^{l-1} \Phi_{2}, \\
& \text { (9) } \quad v\left(I^{(l)}(\underline{\beta})\right) \geq \frac{\Psi_{1}\left(k_{1}\right)}{(4 \Lambda)^{l-1} \Phi_{1}^{l-1}} N\left(\mathcal{P}_{l-1}\right)+\frac{\Psi_{1}\left(k_{1}\right)}{(4 \Lambda)^{l-1}(l-1) \Phi_{1}^{l-2} \Phi_{2}} H\left(\mathcal{P}_{l-1}\right) \text {. }
\end{aligned}
$$

Once more we consider the associated prime ideals $\mathcal{P}^{(1)}, \ldots, \mathcal{P}^{(s)}$ of the ideal $I^{(l)}$ according to Lemma 2, which satisfy

$$
N\left(\mathcal{P}^{(i)}\right) \leq \Phi_{1}^{l}, \quad H\left(\mathcal{P}^{(i)}\right) \leq l \Phi_{1}^{l-1} \Phi_{2} .
$$


If none of the prime ideals $\mathcal{P}^{(i)}, 1 \leq i \leq s$, satisfies (5), from Lemma 2 and (7), (8) we get

$$
\begin{aligned}
& v\left(I^{(l)}(\underline{\beta})\right) \\
& \quad<v(b)+\frac{\Psi_{1}\left(k_{1}\right)}{2(4 \Lambda)^{l-1} \Phi_{1}^{l}} \sum_{i=1}^{s} k_{i} N\left(\mathcal{P}^{(i)}\right)+\frac{\Psi_{1}\left(k_{1}\right)}{2 l(4 \Lambda)^{l-1} \Phi_{1}^{l-1} \Phi_{2}} \sum_{i=1}^{s} k_{i} H\left(\mathcal{P}^{(i)}\right) \\
& \quad \leq \frac{\Psi_{1}\left(k_{1}\right)}{(4 \Lambda)^{l-1} \Phi_{1}^{l-1}} N\left(\mathcal{P}_{l-1}\right)+\frac{\Psi_{1}\left(k_{1}\right)}{(l-1)(4 \Lambda)^{l-1} \Phi_{1}^{l-2} \Phi_{2}} H\left(\mathcal{P}_{l-1}\right),
\end{aligned}
$$

but this contradicts $(9)$. So at least one prime ideal $\mathcal{P}^{\left(i_{0}\right)}$ satisfies $(3)-(5)$, and we choose $\mathcal{P}_{l}=\mathcal{P}^{\left(i_{0}\right)}$.

In the last step for $l=m+1$ the prime ideal $\mathcal{P}_{m} \subset R[y]$ satisfies (3)-(5), and Theorem 2(iii) implies once more

$$
\Psi_{2}(0)<\min \left\{X, V\left(\underline{\beta}, Z\left(\mathcal{P}_{m}\right)\right)\right\} \leq \Psi_{2}\left(k_{1}\right),
$$

so that we can find $k_{m+1} \in\left\{0, \ldots, k_{1}\right\}$ with

$$
\Psi_{2}\left(k_{m+1}\right)<\min \left\{X, V\left(\underline{\beta}, Z\left(\mathcal{P}_{m}\right)\right)\right\} \leq \Psi_{2}\left(k_{m+1}+1\right)
$$

and some $\nu \in\left\{1, \ldots, n_{k_{m+1}}\right\}$ such that $Q_{k_{m+1}}^{(\nu) *} \notin \mathcal{P}_{m}$. Thus Lemma 3 with $r=1$ implies

$$
\begin{aligned}
0 \geq & X / \Lambda-\Phi_{1} H\left(\mathcal{P}_{m}\right)-\Phi_{2} N\left(\mathcal{P}_{m}\right) \\
\geq & \left(\frac{\Psi_{1}\left(k_{1}\right)}{2(4 \Lambda)^{m-1} \Lambda \Phi_{1}^{m}}-\Phi_{2}\right) N\left(\mathcal{P}_{m}\right) \\
& +\left(\frac{\Psi_{1}\left(k_{1}\right)}{2(4 \Lambda)^{m-1} m \Lambda \Phi_{1}^{m-1} \Phi_{2}}-\Phi_{1}\right) H\left(\mathcal{P}_{m}\right),
\end{aligned}
$$

and this completes the proof of Theorem 2 .

4. Proof of Theorem 1. To apply Theorem 2, we begin with the polynomial $Q \in R\left[y_{1}, \ldots, y_{m}\right]$ and define a sequence $\left(Q_{k}\right)_{k \in \mathbb{N}_{0}}$ of polynomials in $R\left[y_{1}, \ldots, y_{m}\right]$ with certain functions $\Phi_{1}, \Phi_{2}, \Psi_{1}, \Psi_{2}: \mathbb{N} \rightarrow \mathbb{R}_{+}$such that

$$
N\left(Q_{k}\right) \leq \Phi_{1}(k), \quad H\left(Q_{k}\right) \leq \Phi_{2}(k), \quad \Psi_{1}(k) \leq v\left(Q_{k}(\underline{\omega})\right) \leq \Psi_{2}(k)
$$

for $k \in \mathbb{N}_{0}$ and $\underline{\omega}=\left(f_{1}(z), \ldots, f_{m}(z)\right)$. Then we choose the parameter $k_{1}$ with respect to $H(Q)$ and $N(Q)$, such that (iii) is satisfied with $\Phi_{1}=\Phi_{1}\left(k_{1}\right)$ and $\Phi_{2}=\Phi_{2}\left(k_{1}\right)$. To fulfill (ii)(d), we notice that $v(\underline{\omega}) \geq 0$, and for each zero $\underline{\theta} \in \overline{C((z))}^{m}$ of the polynomial $Q_{k}$ the inequalities

$$
\begin{aligned}
\Psi_{2}(k) & \geq v\left(Q_{k}(\underline{\omega})\right)=v\left(Q_{k}(\underline{\omega})-Q_{k}(\underline{\theta})\right) \\
& \geq v\left(Q_{k}\right)+v(\underline{\omega}-\underline{\theta}) \geq v(\underline{\omega}-\underline{\theta})
\end{aligned}
$$


hold. Then Theorem 2 yields a bound for $\Psi_{1}\left(k_{1}\right)$ and thereby a bound for $v(Q(\underline{\omega}))=\operatorname{ord}_{0} Q(z, \underline{f}(z))$.

Without loss of generality we suppose that $T(z)=T_{1}(z) / T_{2}(z)$ with $T_{2}(0) \neq 0$, and inductively we define for $k \in \mathbb{N}_{0}$,

$$
\begin{aligned}
& \quad Q_{0}\left(z, y_{1}, \ldots, y_{m}\right)=Q\left(z, y_{1}, \ldots, y_{m}\right) \\
& Q_{k}\left(z, y_{1}, \ldots, y_{m}\right) \\
& =T_{2}(z)^{H\left(Q_{k-1}\right)} A_{0}\left(z, y_{1}, \ldots, y_{m}\right)^{N\left(Q_{k-1}\right)} \\
& \quad \times Q_{k-1}\left(T(z), \frac{A_{1}\left(z, y_{1}, \ldots, y_{m}\right)}{A_{0}\left(z, y_{1}, \ldots, y_{m}\right)}, \ldots, \frac{A_{m}\left(z, y_{1}, \ldots, y_{m}\right)}{A_{0}\left(z, y_{1}, \ldots, y_{m}\right)}\right) .
\end{aligned}
$$

Then for all $k \in \mathbb{N}_{0}$ we have

$$
\begin{gathered}
Q_{k} \in C\left[z, y_{1}, \ldots, y_{m}\right], \quad N\left(Q_{k}\right) \leq t N\left(Q_{k-1}\right) \leq t^{k} N, \\
H\left(Q_{k}\right) \leq d H\left(Q_{k-1}\right)+s N\left(Q_{k-1}\right) \leq d^{k} M+s N \frac{d^{k}-t^{k}}{d-t} \leq \mu M d^{k}
\end{gathered}
$$

with $\mu=1+s /(d-t)$. Since $T_{2}(0) \neq 0$ and $v(T(z))=\delta$, we get for the zero order of

$$
Q_{k}(z, \underline{f}(z))=T_{2}(z)^{H\left(Q_{k-1}\right)} A_{0}(z, \underline{f}(z))^{N\left(Q_{k-1}\right)} Q_{k-1}(T(z), \underline{f}(T(z)))
$$

the bound

$$
\begin{aligned}
\delta \operatorname{ord}_{0} Q_{k-1}(z, \underline{f}(z)) & \leq \operatorname{ord}_{0} Q_{k}(z, \underline{f}(z)) \\
& \leq \delta \operatorname{ord}_{0} Q_{k-1}(z, \underline{f}(z))+N\left(Q_{k-1}\right) \operatorname{ord}_{0} A_{0}(z, \underline{f}(z)),
\end{aligned}
$$

and this implies with $\nu=v(Q(\underline{\omega}))=\operatorname{ord}_{0} Q(z, \underline{f}(z))$,

$\Psi_{1}(k)=\delta^{k} \nu \leq \operatorname{ord}_{0} Q_{k}(z, \underline{f}(z)) \leq \delta^{k} \nu+\frac{\delta^{k}-t^{k}}{\delta-t} N v\left(A_{0}(\underline{\omega})\right) \leq 2 \delta^{k} \nu=\Psi_{2}(k)$,

if we assume without loss of generality that $\nu \geq N v\left(A_{0}(\underline{\omega})\right) /(\delta-t)$. With

$$
\Phi_{1}=N t^{k_{1}}, \quad \Phi_{2}=\mu M d^{k_{1}}, \quad \Lambda=2 \delta, \quad \Psi_{1}(k)=\nu \delta^{k}, \quad \Psi_{2}(k)=2 \nu \delta^{k}
$$

we can apply Theorem 2 . Therefore we choose

$$
k_{1}=\left[\frac{(m-1) \log (8 \delta)+\log (4 m)+m \log N}{\log \delta-m \log t}\right]+1,
$$

and this implies

$$
\nu \delta^{k_{1}} \geq 4 m(8 \delta)^{m-1} \nu N^{m} t^{m k_{1}} .
$$

Now we must distinguish between two cases. If $\Psi_{1}\left(k_{1}\right)$ does not satisfy (iii) of Theorem 2, then

$$
\Psi_{1}\left(k_{1}\right) \leq 2 m^{2}(8 \delta)^{m-1} \Phi_{1}^{m-1} \Phi_{2} \leq m^{2}(8 \delta)^{m} \Phi_{1}^{m} \Phi_{2} .
$$


Otherwise we get the same upper bound from Theorem 2 and deduce

$$
\begin{aligned}
\nu \leq & \mu m^{2}(8 \delta)^{m}\left(d t^{m} \delta^{-1}\right)^{k_{1}} M N^{m} \\
\leq & \mu d \delta^{-1} m^{2}(8 \delta t)^{m}\left(4 m(8 \delta)^{m-1}\right)^{\log d /(\log \delta-m \log t)-1} \\
& \times M N^{m \log d /(\log \delta-m \log t)} .
\end{aligned}
$$

This completes the proof of Theorem 1 .

\section{References}

[B1] P.-G. Becker-Landeck, Maße für algebraische Unabhängigkeit nach einer Methode von Mahler, Acta Arith. 50 (1988), 279-293.

[B2] P.-G. Becker, Effective measures for algebraic independence of the values of Mahler type functions, ibid. 58 (1991), 239-250.

[B3] - Algebraic independence of the values of certain series by Mahler's method, Monatsh. Math. 114 (1992), 183-198.

[B4] - , Transcendence of the values of functions satisfying generalized Mahler type functional equations, J. Reine Angew. Math. 440 (1993), 111-128.

[B5] - , Transcendence measures for the values of generalized Mahler functions in arbitrary characteristic, Publ. Math. Debrecen 45 (1994), 269-282.

[K] K. K. Kubota, Linear functional equations and algebraic independence, in: Transcendence Theory: Advances and Applications, A. Baker and D. W. Masser (eds.), Academic Press, New York, 1977, 227-229.

[L] J. H. Loxton, Automata and transcendence, in: New Advances in Transcendence Theory, A. Baker (ed.), Cambridge Univ. Press, Cambridge, 1988, 215-228.

[LP] J. H. Loxton and A. J. van der Poorten, Transcendence and algebraic independence by a method of Mahler, in: Transcendence Theory: Advances and Applications, A. Baker and D. W. Masser (eds.), Academic Press, New York, 1977, 211-226.

[Ne1] Yu. V. Nesterenko, Estimates for the orders of zeros of functions of a certain class and applications in the theory of transcendental numbers, Izv. Akad. Nauk SSSR Ser. Mat. 41 (1977), 253-284 (in Russian); English transl.: Math. USSR-Izv. 11 (1977), 239-270.

[Ne2] -, On algebraic independence of algebraic powers of algebraic numbers, Mat. Sb. 123 (165) (1984), 435-459 (in Russian); English transl.: Math. USSR-Sb. 51 (1985), 429-454.

[Ne3] - On a measure of the algebraic independence of the values of certain functions, Mat. Sb. 128 (170) (1985), 545-568 (in Russian); English transl.: Math. USSR-Sb. 56 (1987), 545-567.

[Ni1] K. Nishioka, On an estimate for the orders of zeros of Mahler type functions, Acta Arith. 56 (1990), 249-256.

[Ni2] - , Algebraic independence measures of the values of Mahler functions, J. Reine Angew. Math. 420 (1991), 203-214.

[NT] K. Nishioka and T. Töpfer, Transcendence measures and nonlinear functional equations of Mahler type, Arch. Math. (Basel) 57 (1991), 370-378.

[P1] P. Philippon, Critères pour l'indépendance algébrique, Inst. Hautes Etudes Sci. Publ. Math. 64 (1986), 5-52. 
[P2] P. Philippon, Critères pour l'indépendance algébrique dans les anneaux diophantiens, C. R. Acad. Sci. Paris Sér. I Math. 315 (1992), 511-515.

[T1] T. Töpfer, An axiomatization of Nesterenko's method and applications on Mahler functions, J. Number Theory 49 (1994), 1-26.

[T2] - An axiomatization of Nesterenko's method and applications on Mahler functions II, Compositio Math. 95 (1995), 323-342.

[T3] - Algebraic independence of the values of generalized Mahler functions, Acta Arith. 70 (1995), 161-181.

[W] N. C. Wass, Algebraic independence of the values at algebraic points of a class of functions considered by Mahler, Dissertationes Math. 303 (1990).

Am Plattenbusch 48 a

D-51381 Leverkusen

Germany 